\title{
Pixaura: Supporting Tentative Decision Making when Selecting and Sharing Digital Photos
}

\author{
Lalatendu Satpathy, Saara \\ Kamppari, Bridget Lewis \\ HCll, Carnegie Mellon University \\ Pittsburgh, PA 15213-3891 \\ \{lsatpath , skamppar, blewis\} \\ @cs.cmu.edu
}

\author{
Ajay Prasad, Yong Woo Rhee, \\ Benjamin Elgart \\ HCII, Carnegie Mellon University \\ Pittsburgh, PA 15213-3891 \\ \{ajaypras, ywrhee, belgart\} \\ @cs.cmu.edu
}

\author{
Steven Drucker \\ Microsoft Research, Microsoft \\ One Microsoft Way Redmond, WA \\ 98052-6399 \\ sdrucker@microsoft.com
}

\begin{abstract}
Current advances in digital technology promote capturing and storing more digital photos than ever. While photo collections are growing in size, the amount of time that can be devoted to viewing, managing, and sharing digital photos remains constant. Photo decision-making and selection has been identified as key to addressing this concern.

After conducting exploratory research on photo decisionmaking including a wide-scale survey of user behaviors, detailed contextual inquiries, and longer-term diary studies, Pixaura was designed to address problems that emerged from our research. Specifically, Pixaura aims to bridge the gap between importing source photos and sharing them with others, by supporting tentative decision-making within the selection process. For this experience, the system incorporates certain core elements: 1) flexibility to experiment with relationships between photos and groups of photos, 2) the ability to closely couple photos while sharing only a subset of those photos, and 3) a tight connection between the photo selection and photo sharing space.
\end{abstract}

\section{Categories and Subject Descriptors \\ D. 0 [General]}

\section{General Terms}

Design, Human Factors

\section{Keywords}

Digital photos, Selection, Photo triage, Sharing.

\section{INTRODUCTION}

The advent of digital photography has led to an explosion in the number of imported and shared photos that people acquire on their computers. While the capabilities of technology are increasing at a phenomenal rate, human capabilities are not. The amount of time and attention that can be devoted to photo collections is relatively fixed, if not shrinking due to competing demands for users' attention. As Kirk and Sellen point out [9] photowork, the entire process of working with digital photos, has received surprisingly little study. While there has been a great deal of study on digital image display, searching,

(C) The Author 2008.

Published by the British Computer Society browsing, and other aspects of digital photo management, other parts of this digital photo lifecycle have received less attention.

We define the digital photo lifecycle as consisting primarily of three keys stages: selection, authoring of photo experiences, and viewing. This cycle is mainly fueled by the desire to share photos [11] and the stories connected to them with others [4]. We focused on the selection process that we refer to as photo triage, which involves making decisions about individual photos, ranking photos, and determining relationships between groups of photos. The task of selection demands a delicate balance between decisions influenced by the storytelling quality of photos, the method of sharing, and the social relationship with the audience.

Through exploratory research, iterative ideation and evaluation, we have identified that people need support for tentative decision making in the selection process for sharing and have developed a system called Pixaura to address this need. The Pixaura system has been designed to provide a space in which people can leverage the freedom and familiarity of physical photo interactions along with the power and flexibility of the digital realm. Pixaura is a desktop-based photo triage application that integrates into existing photo management and sharing systems, targeted at novice and photo hobbyists.

\section{RELATED WORK}

To give a framework around photo triage, we will highlight the relevant areas from existing digital photo work devoted to photo organization, sharing, interaction, and selection.

\subsection{Photo Organization}

Photo organization has been primarily focused on systems for applying tags or searching based on tags [13], [3], [10]. Tags and other annotations create additional metadata to retrieve photos and create connections between them. Successful retrieval relies largely on people manually attaching appropriate annotations to each photo, since automated image content analysis is still limited [13]. However, studies [3] show that consumers typically put little effort into annotating their photo collections, which can be experienced as work. Recent internet-based experiences such as Flickr have created more compelling reasons for annotation of individual photos since they bring annotation and sharing closer together. They also allow the task of photo annotation to be distributed to friends and the entire Flickr community. When photo organization is more closely coupled with sharing, the user is more likely to engage with their photos to add meaning to them with annotations and create relationships between photos.

\subsection{Photo Sharing}

The most common mediums through which people share their digital photos include e-mail, online albums, personal websites/blogs and sharing in person. The motivations for 
sharing photos are manifold: people share experiences, create awareness, augment communication with visual elements, enhance their social presence, and deepen personal and community relationships [7]. The social aspects, such as the memories and conversation, are of primary importance in photo sharing [13].

The PhotoArcs research found that sharing photos in person was described as the most enjoyable sharing medium, as it is seen as a way of re-creating the past and re-living the experience with others who were present at the time [2].

\subsection{Photo Interaction}

Many systems draw upon physical world analogies to design new interactions with digital documents. These physical analogies are successful with small sets of photos, but tend to have breakdowns when dealing with larger collections. One such system is BumpTop [1] in which a user interacts with files in a virtual three-dimensional space using pen input. We draw heavily from the BumpTop work in the design of the Pixaura system, but tailor the interaction for the more specific task of photo selection and sharing. The drag-and-drop interaction mechanism is natural for photos as it maps to how people hold and move physical photos. This technique was used in prototypes for actions such as photo labeling [10].

The need for both, an overview with thumbnail sized photos and full sized photos for details, has been identified with Zoomable User Interfaces [5], [8]. These systems often focus on using these techniques for large scale photo management, but the same techniques can be adapted for photo selection as well.

\subsection{Photo Selection}

Others have observed the need for work in the photo selection space. Platt et al [11] observed that a common behavior referred to as the funnel effect shows that there are multiple granularities at which people select photos. While people capture many photos, they save a subset of them on their computers. Of these saved photos, they share only selected groups, and print out even smaller subsets. Their selection criteria included good image quality, good composition, and more personal preferences. Selecting and sorting were the most common and time-consuming activities observed among people who participated in research studies [9].

Current systems support absolute inclusion or exclusion from a set. There is no fuzzy ground to make conditional decisions, or to indicate that one photo belongs more to a group than another photo. Our user studies demonstrated a need for such an environment, which we aimed to create with Pixaura.

\section{EXPLORATORY RESEARCH}

In order to gain a better understanding of how people select photos for sharing, we conducted a broad web-based survey with over 300 participants, eight contextual inquiries, four diary studies, and elicited retrospective accounts from ten users. Our focus of these studies was to explore photo selection that takes place in preparation for sharing photo based stories, and how social factors motivate and influence this process. The relevant insights from our research are discussed below.

\subsection{Survey Insights}

In order to confirm preliminary qualitative findings across a larger, more diverse set of people and find new trends in practices, we conducted a survey on the web. The survey was disseminated to a diverse group of people from April $18^{\text {th }}$ to
June $26^{\text {th }}, 2007$. We had 420 individuals respond to the survey, with $300+$ participants responding to all questions.

Sharing quantity: When asked about how frequently people chose different quantities of photos to share, overwhelming number of photo sharers responded that they share only selected photos. $53.5 \%$ of the respondents frequently share only selected photos and $63.5 \%$ never share their entire photo collection. This confirms that the photo triage process is common in our target user base.

Organization Methods: Participants also reported that when they organized and sorted their photos, that they predominantly grouped them by event and by date. They also reported that renaming photo files was not a widespread method for handling them.

\subsection{Contextual Inquiry \& Retrospective Account Insights}

Contextual Inquiries were conducted while each participant interacted with their personal photo collections, either at their homes or at their work-places. The insights from each interview were modeled individually, and later consolidated into flow and cultural models. Insights from the retrospective accounts were incorporated into the consolidated models.

Subjective Decision Criteria: None of our participants could explain to us how they made certain decisions about their photos. Many participants used the term "best" when selecting photos, and mentioned photo quality, how interesting it was, how good the people in it looked, or rarity of that kind of photo when asked to explain what "best" meant. From our observations, photo selection relies heavily on emotional and contextual connections to photos that are not inherent in the photo alone.

Lack of Triage Support: There is a distinct lack of support for recording users' decisions and thoughts about photos during the photo triage process. We observed the use of external tools, such as text editors, to write notes about which photos they have selected, as well as participants keeping track of their decisions in their mind. Users did not want to move pictures from their original location since they would lose the context provided by their photo management environment.

Multiple Photos and Passes: Most of our users took multiple photos of the same subject, and later chose the best shot on the computer. One user said "I keep taking pictures until I get a good one." The funnel effect [9] was observed, in conjunction with the user taking multiple passes through a photo collection.

\subsection{Diary Study Insights}

To further understand peoples' practices and behaviors over time, we gained insight from four diary study participants. The diaries consisted of questions about their behaviors at every point in the digital photo lifecycle. These questions were to be answered whenever the participants dealt with digital photos.

In short sessions of photowork which involved working with digital; participants recorded various activities including acquiring, editing, organizing and sharing digital photos. Upon reflection on her use of time, one participant wrote that she had yet to learn efficient practices to edit, manage, and share her photos, offering, "I'm still floundering around." Mostly this was because, as she said; "I feel rushed much of the time and haven't studied the programs that I am using even now." Yet she also wrote that she wanted to "spend more time with [her] pictures." From the diary studies, we identified that users 
wanted to spend less time managing their photos and instead spend their time directly interacting with their photos.

\section{DESIGN IMPLICATIONS}

The following design implications are based on a synthesis of prior related work, our exploratory research, and iterative ideation and evaluation phases, and are core to Pixaura.

Flexibility: Give users the flexibility to experiment with relationships between photos and groups of photos. With a loose structure and utilization of spatial relationships, users have the ability to create connections between photos or group of photos, without having to be explicit about them such as placing them in files or even albums. The freedom and flexibility of physical photo interaction can be merged with the advantages of digital space, such as copying photos and space manipulation.

Tentative Decisions: As users make tentative decisions, give them the ability to closely couple photos with varying levels of inclusion. As users refine their photo selections, potentially for multiple audiences, they need to be able to express different levels and types of decisions.

Bridge the Gap: Provide a tight connection between the photo selection and photo sharing space. By allowing photo triage and sharing to occur in a single space, users can attend to the sharing task without having to switch application context. New goals that arise while pursuing an initial goal, such as finding a photo to email to mom while selecting photos to add to a webalbum shared with friends, can also be attended to when selection and sharing are integrated within the same space.

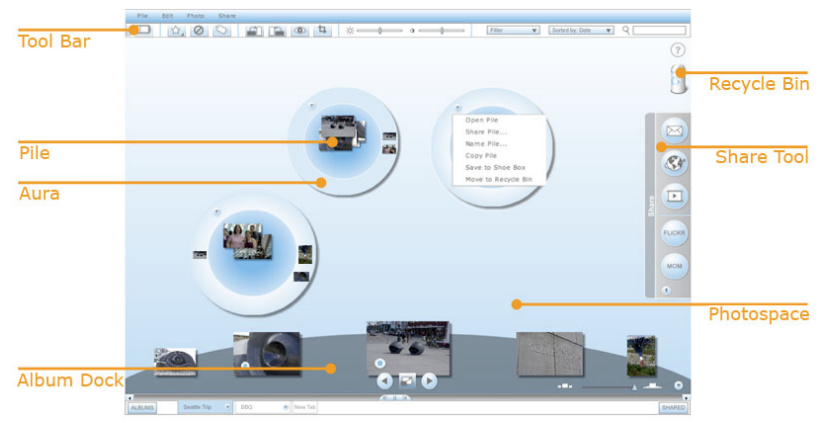

Figure 1. Multiple Piles and Album Dock

\section{PIXAURA}

The three main components of Pixaura are the album dock, the photo piles with auras, and the share tool bar. The album dock is where the source photos are held accessible for use. The main interaction in Pixaura centers on creating and altering the placement of photos from the album dock into various photo piles and their associated auras. These piles are then shared directly with friends and family using the share toolbar.

\subsection{Photo Piles}

Dragging a photo from the album dock to the main space, referred to as the photospace, creates a new photo pile. The pile is given structure with a two-tiered circle, centered around the given photo. The circle was chosen for the playful and organic feel it created. More photos can be placed in the center of the pile, or they can be placed in the outer-tier, known as the aura of the pile.

\subsection{Pile Auras}

The aura allows for users to associate photos with a pile, without having to add them to the pile itself. This supports users in making tentative decisions. This initial version of
Pixaura only allows for one tier of auras and tentative decisions, and further research can explore the optimal number of tiers. When sharing piles, the photos in the aura are not shared, so only the photos the user has placed in the center of the pile are seen by others. In this way, the aura also allows for privacy and ranking of photos, as only the ones chosen as public or best of a group are shared. While a global star-based ranking system requires a user to decide what each level means for all instances, this local ranking can have different parameters for every pile. The two-tiered pile also allows users to share different selections of photos from the pile, while keeping the same group of photos together. We limited the pile to two tiers to explore how users interact with a structure that provides fuzzy associations.

The piles combine the flexibility found in physical photo interaction with the benefits of digital space; photos can be easily placed in multiple piles, something that would be difficult in a non-digital space. The piles themselves can also be moved in the photospace to create spatial relationships, or be minimized when not being used. Pile labels can be added and edited directly on the title bar of an opened pile, or at any time through the pile options menu.

\subsection{Album Dock}

When a photo is dragged from the album dock into piles, a linked copy of that photo is created in the photospace, and the photo in the dock is marked as being placed in a pile. The album is kept intact in order to preserve the original context from which the piled photos come. The preservation of the original folders and albums gives users a sense of security to manipulate them within the piles in the photospace, knowing that they can always return to or refer back to the original.

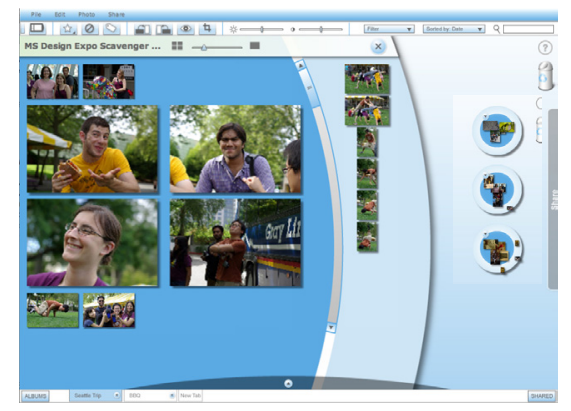

Figure 2. Open Pile with Minimized Piles

\subsection{Refining Decisions}

To assist users in refining selections, they can open a pile to look through and reorder the photos in a grid view. The grid has a fisheye display that provides a larger photo for the users' focus. The photos can be moved between the center and aura of the pile, as well as to the other piles, minimized at the side of the photospace. Manipulations such as tagging and cropping can be made to individual or groups of photos. Users can also enlarge photos from both the center and aura to compare photos side-by-side, and switch back and forth between photos to make fine tuning decisions. 


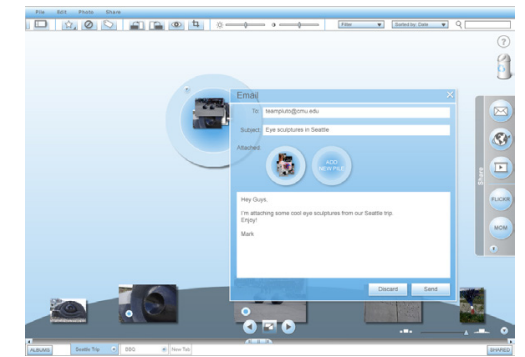

Figure 3. Sharing a Pile through E-mail

\subsection{Sharing Portals}

To share, users can drag a pile to a portal on the share tool bar. The standard portals consist of the most common digital photo sharing mediums; email, web, and a presentation mode for sharing in person. In addition, users can create custom portals to frequent recipients or websites, and edit them as social demands change. Multiple piles can be shared during one sharing session. These share portals bridge the gap between organizing and sharing photos, making it easy to stay socially connected through digital photos.

\section{USER REACTION TO THE SYSTEM}

While a formal study of Pixaura was beyond the scope of the current work, we did a casual evaluation of the system with a Think-aloud user study. Participants were novice photographers, who were given one-time-use digital cameras to capture photos to use in the Pixaura system. The 10 participants, ages 23-60, carried out two sets of tasks with no prior training. The first set of tasks was meant to familiarize them with Pixaura, while a second set of tasks consisted primarily of the participants using Pixaura to explore and share a set of their own digital photos for the first time.

Intuitive and Organic: [AP3] When using Pixaura, participants found the drag-and-drop interface familiar and easy to use. Confusion regarding the creation of piles in an early prototype was overcome by providing stronger visual feedback. The visual language, consisting of curves and animated transitions, created a smooth flow as users experienced the system.

One participant commented that Pixaura will encourage people to take more photos. Seeing the already created piles would remind users of things to take photos of, which then they can add to previously created piles.

Understanding Piles and Auras: To test if participants would figure out the pile and aura structure on their own, we did not explain their purpose beforehand. Most participants did not pay attention to the aura until it was explained to them in between tasks. This is likely because the concept of a conditional decision in a digital space was unfamiliar to them.

While a few participants used the aura as it was designed for, others took advantage of its loosely defined purpose. One participant created a group of the best photos in a pile and then placed all the photos of her cat in the aura, because "no one else wants to see photos of [her] cat."

From our think-aloud studies, we saw that the piles tend to form either theme based groups, or recipient based groups. The amount of piles created during the tasks was small, with no more than four in the photospace at a time. To a degree, this small amount was caused by a constraint in the number of source photos the participants had. However, it is feasible that for a source album from a single event, a user will create a limited amount of piles based on theme and recipient.

\section{CONCLUSION}

The Pixaura system was designed based on a variety of user research to address an unmet need for supporting the tentative decision making nature of photo selection.

By using a familiar, physical metaphor for moving photos around a physical space, novices were easily able to use the system. Allowing 'fuzzy' membership into groups through the 'aura' around a pile matched user requests to help deal with ambiguity. Tying selection and sharing closely together was key to helping users have a fluid experience within the digital photo lifecycle.

\section{FUTURE DIRECTIONS}

There are further insights from our research and design that were out of scope for the timeline of this project. We identified several areas of interest within photo triage, which we encourage others to explore. 1) Creating connections, and revealing underlying connections, can help users navigate their photo collections. Using visualizations such as weight, and results of photo sharing could show the different ways photos are related to each other can help users search for photos they are communicating stories about. Connecting groups of photos together can create meaningful associations that assist users in understanding their photo collection. In Pixaura, these group connections could be made by providing users the ability to partially combine piles, creating a link with their auras. 2) When photo sharers do not take the time to triage their photos, but instead "dump" all of their photos into a shared repository, the audience is left with the burden of selecting the photos to find those that interest them. There is a great opportunity here to assist selectively viewing received photos, while addressing how this will influence social relationships between the sharer and the audience.

\section{REFERENCES}

[1] Agarawala, A. and Balakrishnan, R. 2006. Keepin' it real: pushing the desktop metaphor with physics, piles and the pen. In Proc. of CHI '06, 1283-1292.

[2] Ames, M. and Manguy, L. 2006. PhotoArcs: a tool for creating and sharing photo-narratives. In Proc. of the CHI '06, 466-471.

[3] Ames, M. and Naaman, M. 2007. Why we tag: motivations for annotation in mobile and online media. In Proc. of the SIGCHI. CHI '07. 971-980.

[4] Balabanović, M., Chu, L. L., and Wolff, G. J. 2000. Storytelling with digital photographs In Proc. of the CHI'00, 564-571.

[5] Bederson, B. B. 2001. PhotoMesa: a zoomable image browser using quantum treemaps and bubblemaps. In Proc. of UIST'01, 71-80.

[6] Davis, M., Van House, N., Towle, J., King, S., Ahern, S., Burgener, C., Perkel, D., Finn, M., Viswanathan, V., and Rothenberg, M. 2005. MMM2: mobile media metadata for media sharing. In CHI '05, 1335-1338

[7] Frohlich, D., Kuchinsky, A., Pering, C., Don, A., and Ariss, S. 2002. Requirements for photoware CSCW '02, 166-175.

[8] Huynh, D. F., Drucker, S. M., Baudisch, P., and Wong, C. 2005. Time quilt: scaling up zoomable photo browsers for large, unstructured photo collections. In the Proc. of CHI '05, 1937-1940. 
[9] Kirk, D., Sellen, A., Rother, C., and Wood, K. 2006. Understanding photowork. In Proc. of the CHI '06, 761770 .

[10] Kuchinsky, A., Pering, C., Creech, M. L., Freeze, D., Serra, B., and Gwizdka, J. 1999. FotoFile: a consumer multimedia organization and retrieval system. In the Proc. of $\mathrm{CHI}$ '99., 496-503
[11] Platt, John C. AutoAlbum: Clustering Digital Photographs using Probabalistic Model Merging, Proc. of the IEEE Workshop on CBAIVL '00, pp. 96, June 2000.

[12] Qian, Y. and Feijs, L. M. 2004. Exploring the potentials of combining photo annotating tasks with instant messaging fun. In Proc. of the MUM '04, vol. 83., 11-17.

[13] Shneiderman, B., Bederson, B. B., and Drucker, S. M. 2006. Find that photo!: interface strategies to annotate, browse, and share. Commun. ACM 49, 4 (Apr. 2006), 69- 